FACTA UNIVERSITATIS (NIŠ)

Ser. Math. Inform. Vol. 35, No 2 (2020), 541-547

https://doi.org/10.22190/FUMI2002541J

\title{
ON THE SIGNED MATCHINGS OF GRAPHS
}

\author{
Samane Javan and Hamid Reza Maimani*
}

(C) 2020 by University of Niš, Serbia | Creative Commons Licence: CC BY-NC-ND

\begin{abstract}
For a graph $G$ and any $v \in V(G), E_{G}(v)$ is the set of all edges incident with $v$. A function $f: E(G) \rightarrow\{-1,1\}$ is called a signed matching of $G$ if $\sum_{e \in E_{G}(v)} f(e) \leq 1$ for every $v \in V(G)$. The weight of a signed matching $f$, is defined by $w(f)=\sum_{e \in E(G))} f(e)$. The signed matching number of $G$, denoted by $\beta_{1}^{\prime}(G)$, is the maximum $w(f)$ where the maximum is taken over all signed matchings over $G$. In this paper, we have obtained the signed matching number of some families of graphs and studied the signed matching number of subdivision and the edge deletion of edges of a graph.
\end{abstract}

Keywords: signed matching; signed matching number; bipartite graphs.

\section{Introduction}

In this paper, $G$ is a simple graph with vertex set $V=V(G)$ and edge set $E=E(G)$. The order $|V|$ and size $|E|$ of $G$ is denoted by $n(G)$ and $m(G)$, respectively.

Let $G=(V, E)$ be a graph. For $u \in V, E_{G}(v)=\{u v \in E \mid u \in V\}$ are called the edgeneighborhood of $v$ in $G$. For simplicity $E_{G}(v)$ is denoted by $\mathrm{E}(v)$. The degree of a vertex $v \in V$ is $\operatorname{deg}_{G}(v)=d_{G}(v)=|E(v)|$. The minimum degree and the maximum degree of a graph $G$ are denoted by $\delta=\delta(G)$ and $\Delta=\Delta(G)$, respectively. A vertex of degree one is called a leaf and its neighbour is called a support vertex. A graph, $G$, is called $r$-regular graph if $\operatorname{deg}_{G}(v)=r$ for every $v \in V(G)$. For a nonempty subset $X \subseteq E$ the edge induced subgraph of $G$, induced by $X$, denote by $\langle X\rangle$, is a subgraph with edge set $X$ and a vertex $v$ belong to $\langle X\rangle$ if $v$ is incident with at least one edge in $X$. A $k$-partite graph is a graph which its vertex set can be partitioned into $k$ sets $V_{1}, V_{2}, \cdots, V_{k}$ such that every edge of the graph has an end point in $V_{i}$ and an end point in $V_{j}$ for some $1 \leq i \neq j \leq k$. A complete $k$-partite graph is a $k$-partite graph that every vertex of each partite set is adjacent to all vertices of the other partite sets. We denote the complete $k$-partite graph

Received December 31, 2019; accepted January 26, 2020

2010 Mathematics Subject Classification. Primary 05C69

* Corresponding Author 
by $K_{n_{1}, n_{2}, \cdots, n_{k}}$, where $\left|V_{i}\right|=n_{i}$ for $1 \leq i \leq k$. In the case $k=2$, the $k$-partite and complete $k$-partite graph are called bipartite and complete bipartite graphs. We denote by $P_{n}, C_{n}, K_{n}$ and $\overline{K_{n}}$, the path, the cycle, complete graph and the empty graph of order $n$, respectively. A double star $D S_{a, b}$ is a graph containing exactly two non-leaf vertices which one is adjacent to $a$ leaves and the other is adjacent to $b$ leaves. These two non-leaf of double star are called centers of double star. For a graph $G=(V, E)$ and $e=u v \in E$, a subdivision of $G$ respect to $e$, denote by $S(G)$, is a graph obtained from $G$ by deleting the edge $e$ and add new vertex $x$ and new edges $x u$ and $x v$. Let $G_{1}=\left(V_{1}, E_{1}\right)$ and $G_{2}=\left(V_{2}, E_{2}\right)$ be two disjoint vertex sets. A graph $G=(V, E)$ is the join graph of $G_{1}$ and $G_{2}$, if $V=V_{1} \cup V_{2}$ and $E=E_{1} \cup E_{2} \cup\left\{u v: u \in V_{1}, v \in V_{2}\right\}$. If $G$ is the join graph of $G_{1}$ and $G_{2}$, we shall write $G=G_{1}+G_{2}$. The graphs $W_{n}=C_{n}+K_{1}, F_{n}=P_{n}+K_{1}$, and $F r_{n}=n K_{2}+K_{1}$ are called wheel, fan and friendship graphs, respectively. For all graph-theoretic terminology not defined here, the reader is referred to [2].

Let $f: E(G) \rightarrow\{-1,1\}$ be a function. For every vertex $v$, we define $f_{G}(v)=$ $\sum_{e \in E_{G}(v)} f(e)$. A function $f: E(G) \rightarrow\{-1,1\}$ is called a signed matching of $G$ if $f_{G}(v) \leq 1$ for every $v \in V(G)$. The weight of a signed matching $f$ is defined by $w(f)=f(E(G))=\sum_{e \in E(G)} f(e)$. The signed matching number of $G$ is $\beta_{1}^{\prime}(G)=\max$ $w(f)$, where the maximum is taken over all signed matchings. It seems natural to define $\beta_{1}^{\prime}\left(\overline{K_{n}}\right)=0$ for all totally disconnected graphs $\overline{K_{n}}$. A signed matching $f$ on $G$, with $w(f)=\beta_{1}^{\prime}(G)$ is called a $\beta_{1}^{\prime}$ - signed matching.

The concept of signed matching is defined by Wang [4], and further studied in, for example $[3,5,6]$. In [4], it is shown that a maximum signed matching can be found in strongly polynomial time. In addition, the exact value of $\beta_{1}^{\prime}(G)$ for paths, cycles, complete graphs and complete bipartite graphs were found [4].

In this paper, we have studied the signed matchings of subdivision and edge deletion of a graph. Also, we have studied the signed matchings of join of graphs.

\section{Main Results}

In this section, we first stated some of the results which would be useful in the remaining part of the paper. The following proposition provides a relation between $|E(G)|$ and $\beta_{1}^{\prime}(G)$.

Proposition 2.1. For any graph $G=(V(G), E(G))$, we have $\beta_{1}^{\prime}(G) \equiv|E(G)|(\bmod 2)$.

Proof. Let $f$ be a $\beta_{1}^{\prime}$-signed matching on $G$. Suppose that $P$ and $M$ are the numbers of positive and negative edges respect to $f$, respectively. Hence

$$
P+M=|E(G)|, P-M=\beta_{1}^{\prime}(G) .
$$

Therefore, $\beta_{1}^{\prime}(G)-|E(G)|=-2 M$ and we conclude that $\beta_{1}^{\prime}(G) \equiv|E(G)|(\bmod 2)$.

In [4], $\beta_{1}^{\prime}(G)$ for Eulerian graphs is given as follows. 
Theorem 2.1. [4] Let $G$ be a Eulerian graph of order $n$ and size $m$. Then

$$
\beta_{1}^{\prime}(G)=\frac{1}{2}\left((-1)^{m}-1\right) .
$$

Corollary 2.1. [4]Let $n$ be a natural number. Then

$$
\beta_{1}^{\prime}\left(C_{n}\right)= \begin{cases}-1, & \text { if } n \neq 2 k \\ 0, & \text { if } n=2 k\end{cases}
$$

For non-Eulerian graph, the following theorem was given in [4]. Here we give an alternative proof for this theorem.

Theorem 2.2. Let $G$ be a graph of order $n$ with $2 k(k \geq 1)$ odd vertices. Then

$$
0 \leq \beta_{1}^{\prime}(G) \leq k .
$$

Proof. Let $f: E(G) \longrightarrow\{1,-1\}$ be a $\beta_{1}^{\prime}$-signed matching of $G$. Hence $f_{G}(v) \leq 0$ for any even vertex $v$ and $f_{G}(v) \leq 1$ for any odd vertex $v$. Therefore

$$
\left.2 \beta_{1}^{\prime}(G)=2 \sum_{e \in E} f(e)=\sum_{v \in V} f_{G}(v)\right) \leq 2 k
$$

and hence $\beta_{1}^{\prime}(G) \leq k$.

For the lower bound, note that, the edges of $G$ can be partitioned to subsets $E_{1}, E_{2}, \cdots E_{k}$, such that for each $i$, the induced subgraph $\left\langle E_{i}\right\rangle$ is a trail connected odd vertices and at most one of these trails has odd length (see Theorem 5.3 of [2]). If we label the edges of each $E_{i}$ alternately by 1 and -1 , we can find a signed matching with positive weight. Hence $\beta_{1}^{\prime}(G) \geq 0$.

As a straight result of Theorems 2.1 and 2.2, we have the following corollary.

Corollary 2.2. Let $G$ be a graph. Hence $\beta_{1}^{\prime}(G)=-1$ if and only if $G$ is a Eulerian graph of odd size.

Theorem 2.3. [4] Let $m$ and $n$ be two natural numbers. Then

$$
\beta_{1}^{\prime}\left(K_{m, n}\right)= \begin{cases}0 & \text { if } m n \equiv 0(\bmod 2), \\ \min \{m, n\} & \text { if } m n \equiv 1(\bmod 2) .\end{cases}
$$

Theorem 2.4. Let $m, n, p$ be positive integers. Then

$$
\beta_{1}^{\prime}\left(K_{m, n, p}\right)= \begin{cases}0 & \text { if } m \equiv n \equiv p \equiv 0(\bmod 2) \\ -1 & \text { if } m \equiv n \equiv p \equiv 1(\bmod 2), \\ 0 & \text { if } m \equiv n \equiv 0(\bmod 2), p \equiv 1(\bmod 2), \\ \min \{m, n\} & \text { if } m \equiv n \equiv 1(\bmod 2), p \equiv 0(\bmod 2)\end{cases}
$$


Proof. If $m \equiv n \equiv p(\bmod 2)$, then each vertex of $K_{m, n, p}$ has even degree and hence $K_{m, n, p}$ is an Eulerian graph. Therefore, the first and the second parts of theorem are obtained by Theorem 2.1. Now suppose that $V_{1}=\left\{v_{1}, v_{2}, \ldots, v_{m}\right\}, V_{2}=$ $\left\{u_{1}, u_{2}, \ldots, u_{n}\right\}$ and $V_{3}=\left\{w_{1}, w_{2}, \ldots w_{p}\right\}$ are three parts of $K_{m, n, p}$ of sizes $m, n$ and $p$, respectively. Let $f: E(G) \longrightarrow\{1,-1\}$ be a signed matching of $K_{m, n, p}$. At first consider the case $m \equiv n \equiv 0(\bmod 2)$ and $p \equiv 1(\bmod 2)$. Hence every vertex of $V_{3}$ has even degree. Therefore $f_{K_{m, n, p}}(v) \leq 0$ for any $v \in V_{3}$. On the other hand $K_{m, n, p} \cong K_{m+n, p} \cup K_{m, n}$ and hence

$$
w(f)=\sum_{v \in V_{3}} f_{K_{m, n, p}}(v)+\sum_{v \in V_{2}} f_{K_{m, n}}(v) .
$$

Note that For any $v \in V_{2}$, the degree of $v$ in $K_{m, n}$ is even and hence $f_{K_{m, n}}(v) \leq$ 0 . Therefore $w(f) \leq 0$. Hence $\beta_{1}^{\prime}\left(K_{m, n, p}\right) \leq 0$. Now consider the function $g$ : $E\left(K_{m, n, p}\right) \longrightarrow\{1,-1\}$ as follows:

$$
g\left(u_{i} v_{j}\right)=(-1)^{i+j}, g\left(u_{i} w_{j}\right)=(-1)^{i+j}, g\left(w_{i} v_{j}\right)=(-1)^{i+j} .
$$

It is not difficult to see that $g$ is a signed matching and $w(g)=0$. Therefore,, in this case $\beta_{1}^{\prime}\left(K_{m, n, p}\right)=0$.

Now suppose that $m \equiv n \equiv 1(\bmod 2)$ and $p \equiv 0(\bmod 2)$. Again, every vertex of $V_{3}$ has even degree. Therefore $f_{K_{m, n, p}}(v) \leq 0$ for any $v \in V_{3}$. By the same argument as above we have

$$
w(f)=\sum_{v \in V_{3}} f_{K_{m, n, p}}(v)+f\left(E\left(K_{m, n}\right)\right) \leq f\left(E\left(K_{m, n}\right)\right) .
$$

But $f\left(E\left(K_{m, n}\right)\right) \leq \min \{m, n\}$ by Theorem 2.3. Hence $\beta_{1}^{\prime}\left(K_{m, n, p}\right) \leq \min \{m, n\}$ By the same argument as above $\beta_{1}^{\prime}\left(K_{m, n, p}\right)=\min \{m, n\}$.

Theorem 2.5. Suppose that $a$ and $b$ are two integers. Then

$$
\beta_{1}^{\prime}\left(D S_{a, b}\right)= \begin{cases}3 & \text { if } a \equiv b \equiv 0(\bmod 2) \\ 1 & \text { if } a \equiv b \equiv 1(\bmod 2) \\ 2 & \text { if } a \equiv 1(\bmod 2), b \equiv 0(\bmod 2)\end{cases}
$$

Proof. Let $u$ and $v$ be centers of double star $D S_{a, b}$ of degrees $a+1$ and $b+1$. Suppose that $f: E\left(D S_{a, b}\right) \longrightarrow\{1,-1\}$ is a signed matching set. Hence $w(f)=$ $f_{D S_{a, b}}(u)+f_{D S_{a, b}}(v)-f(u v)$.

If $a \equiv b \equiv 1(\bmod 2)$, then $\operatorname{deg}(u)$ and $\operatorname{deg}(v)$ are even. Therefore, it follows that $f_{D S_{a, b}}(u), f_{D S_{a, b}}(v) \leq 0$. We conclude $w(f) \leq-f(u v) \leq 1$. Hence $\beta_{1}^{\prime}\left(D S_{a, b}\right) \leq$ 1. Now consider $g: E\left(D S_{a, b}\right) \longrightarrow\{1,-1\}$ such that $g(e)=1$ for $\frac{a+1}{2}$ edges of $E_{D S_{a, b}}(v) \backslash\{u v\}$ and $\frac{b+1}{2}$ edges of $E_{D S_{a, b}}(u) \backslash\{u v\}$ and $g(e)=-1$ for the remaining edges of $E_{D S_{a, b}}(v) \cup E_{D S_{a, b}}(u)$. Clearly $g$ is a signed matching and $w(g)=1$. Hence $\beta_{1}^{\prime}\left(D S_{a, b}\right) \geq 1$ and we conclude $\beta_{1}^{\prime}\left(D S_{a, b}\right)=1$.

If $a \equiv b \equiv 0(\bmod 2)$, then $\operatorname{deg}(u)$ and $\operatorname{deg}(v)$ are odd. Therefore, it follows $\left.f_{D S_{a, b}}(v)\right), f_{D S_{a, b}}(u) \leq 1$. We conclude that $w(f) \leq 2-f(u v) \leq 3$. Hence 
$\beta_{1}^{\prime}\left(D S_{a, b}\right) \leq 3$. Now consider $g: E\left(D S_{a, b}\right) \longrightarrow\{1,-1\}$ such that $g(e)=1$ for $\frac{a+2}{2}$ edges of $E_{D S_{a, b}}(v) \backslash\{u v\}$ and $\frac{b+2}{2}$ edges of $E_{D S_{a, b}}(u) \backslash\{u v\}$ and $g(e)=-1$ for the remaining edges of $E(v) \cup E(u)$. Again $g$ is a signed matching with $w(g)=3$ and we conclude that $\beta_{1}^{\prime}\left(D S_{a, b}\right)=3$.

For the last case, suppose that $a \equiv 0(\bmod 2)$ and $b \equiv 1(\bmod 2)$. By the same argument as above, we conclude that $\beta_{1}^{\prime}\left(S_{a, b}\right)=2$.

Theorem 2.6. Let $n$ be an integer. Then

$$
\beta_{1}^{\prime}\left(W_{n}\right)= \begin{cases}\left\lfloor\frac{n+1}{2}\right\rfloor & \text { if } n \equiv 0,3(\bmod 4), \\ \left\lfloor\frac{n+1}{2}\right\rfloor-1 & \text { if } n \equiv 1,2(\bmod 4) .\end{cases}
$$

Proof. Suppose that $E\left(W_{n}\right)=\left\{v_{i} v_{i+1}, u v_{i}: 1 \leq i \leq n\right\}$, where indices computing in module $n$. Note that the vertex $u$ has degree equal to $n$, and other vertices have degree 3 . If $n \equiv 0(\bmod 4)$, then $W_{n}$ has $n$ vertices of odd degree. Hence $\beta\left(W_{n}\right) \leq \frac{n}{2}=\left\lfloor\frac{n+1}{2}\right\rfloor$ by Theorem 2.2. Now define $f: E\left(W_{n}\right) \longrightarrow\{1,-1\}$ by

$$
f\left(u v_{4 i+1}\right)=f\left(u v_{4 i+2}\right)=f\left(v_{4 i+2} v_{4 i+3}\right)=f\left(v_{4 i+3} v_{4 i+4}\right)=f\left(v_{4 i+4} v_{4 i+5}\right)=1
$$

for $0 \leq i \leq \frac{n}{4}-1$ and $f(e)=-1$ for other edges of $W_{n}$. Clearly $f$ is a signed matching with $w(f)=\frac{n}{2}=\left\lfloor\frac{n+1}{2}\right\rfloor$. So $\beta_{1}^{\prime}\left(W_{n}\right) \geq\left\lfloor\frac{n+1}{2}\right\rfloor$. Hence $\beta_{1}^{\prime}\left(W_{n}\right)=\left\lfloor\frac{n+1}{2}\right\rfloor$. The case $n \equiv 3(\bmod 4)$ is obtained by a similar argument as the above.

Now suppose that $n \equiv 2(\bmod 4)$. Hence $\beta_{1}^{\prime}\left(W_{n}\right) \leq \frac{n}{2}$ by Theorem 2.2. But $\beta_{1}^{\prime}\left(W_{n}\right) \neq \frac{n}{2}$ by Proposition 2.1 and therefore $\beta_{1}^{\prime}\left(W_{n}\right) \leq \frac{n}{2}-1$. Now define $f$ : $E\left(W_{n}\right) \longrightarrow\{1,-1\}$ by

$$
\begin{gathered}
f\left(u v_{n-1}\right)=f\left(v_{1} v_{n}\right)=1, \\
f\left(u v_{4 i+1}\right)=f\left(u v_{4 i+2}\right)=f\left(v_{4 i+2} v_{4 i+3}\right)=f\left(v_{4 i+3} v_{4 i+4}\right)=f\left(v_{4 i+4} v_{4 i+5}\right)=1
\end{gathered}
$$

for $0 \leq i \leq \frac{n-6}{4}$ and $f(e)=-1$ for other edges of $W_{n}$. Clearly $f$ is a signed matching with $w(f)=\frac{n}{2}-1=\left\lfloor\frac{n+1}{2}\right\rfloor-1$. So $\beta_{1}^{\prime}\left(W_{n}\right) \geq\left\lfloor\frac{n+1}{2}\right\rfloor-1$.

Theorem 2.7. Let $n$ be an integer. Then

$$
\beta_{1}^{\prime}\left(F_{n}\right)= \begin{cases}\left\lfloor\frac{n-1}{2}\right\rfloor-1 & \text { if } n \equiv 0,3(\bmod 4), \\ \left\lfloor\frac{n-1}{2}\right\rfloor & \text { if } n \equiv 1,2(\bmod 4) .\end{cases}
$$

Proof. The result follows by a similar argument as the proof of Theorem 2.6.

Theorem 2.8. Let $n$ be an integer. Then

$$
\beta_{1}^{\prime}\left(F r_{n}\right)= \begin{cases}0 & \text { if } n \equiv 0(\bmod 2) \\ -1 & \text { if } n \equiv 1(\bmod 2)\end{cases}
$$

Proof. Since the graph $\mathrm{Fr}_{n}$ is an Eulerian graph, the result follows from Theorem 2.1. 
Theorem 2.9. Let $G$ be a graph and e be an edge of $G$. If $S(G)$ is the subdivision of $G$ by edge e, then

$$
\beta_{1}^{\prime}(G)-1 \leq \beta_{1}^{\prime}(S(G)) \leq \beta_{1}^{\prime}(G)+1 .
$$

In addition these bounds are sharp.

Proof. Suppose that $e=u v$ and $S(G)=G \backslash\{e\} \cup\{x u, x v\}$, where $x$ is the new vertex. Let $f$ be a $\beta_{1}^{\prime}$-signed matching of $G$. If $f(e)=1$ (or $f(e)=-1$ ), then define $g: E(S(G)) \longrightarrow\{1,-1\}$ by $g(x u)=1$ (or $g(x u)=-1), g(x v)=-1$ and $g(w)=f(w)$ for other edges of $S(G)$. Clearly $g$ is a signed matching on $S(G)$ and $w(g)=\beta_{1}^{\prime}(G)-1$. Hence $\beta_{1}^{\prime}(G)-1 \leq \beta_{1}^{\prime}(S(G))$.

Now suppose that $f$ is a $\beta_{1}^{\prime}$-signed matching of $S(G)$. Define signed matching $g$ on $G$ by $g(e)=-1$ and $g(w)=f(w)$ for other edges of $G$. we conclude that $\beta_{1}^{\prime}(S(G)) \leq \beta_{1}^{\prime}(G)+1$.

For any positive integer $n$, we have $S\left(C_{n}\right)=C_{n+1}$. If $n$ is even, then $\beta_{1}^{\prime}\left(C_{n}\right)=0$ and $\beta_{1}^{\prime}\left(C_{n+1}\right)=-1$ by Corollary 2.1 and the lower bound is occurred. If $n$ is odd, then $\beta_{1}^{\prime}\left(C_{n}\right)=-1$ and $\beta_{1}^{\prime}\left(C_{n+1}\right)=0$ by Corollary 2.1 and the we obtain the upper bound.

Theorem 2.10. Let $G$ be a graph. Then

$$
\beta_{1}^{\prime}(G)-3 \leq \beta_{1}^{\prime}(G-e) \leq \beta_{1}^{\prime}(G)+1 .
$$

In addition these bounds are sharp.

Proof. Suppose that $e=u v$. Let $f$ be a $\beta_{1}^{\prime}$-signed matching of $G$. If $f(e)=1$, then define $g: E(G-e) \longrightarrow\{1,-1\}$ by $g(x)=f(x)$ for any edge $x$ of $G-e$. Clearly $g$ is a signed matching on $G-e$ and $w(g)=\beta_{1}^{\prime}(G)-1$. Hence $\beta_{1}^{\prime}(G)-1 \leq \beta_{1}^{\prime}(G-e)$. If $f(e)=-1$, change the label of two edges $e_{1}$ and $e_{2}$ (which are adjacent to $u$ and $v$ in $G-e$, respectively) from 1 to -1 . Hence we have a signed matching on $G-e$ of weight $\beta_{1}^{\prime}(G)-3$ and hence $\beta_{1}^{\prime}(G)-3 \leq \beta_{1}^{\prime}(G-e)$.

Now suppose that $f$ is a $\beta_{1}^{\prime}$-signed matching of $G-e$. Define signed matching $g$ on $G$ by $g(e)=-1$ and $g(w)=f(w)$ for other edges of $G$. We conclude that $\beta_{1}^{\prime}(G-e) \leq \beta_{1}^{\prime}(G)+1$.

Suppose that $n$ is an even integer. We have $\beta_{1}^{\prime}\left(D S_{n, n}\right)=3$ by Theorem2.5. If $x, y$ are centers of double star and $e=x y$, then $D S_{n, n}-e=2 K_{1, n}$ and we have $\beta_{1}^{\prime}\left(D S_{n, n}-e\right)=0$ by Theorem 2.3. Hence the lower bound is obtained. If $n$ is even and $m$ is odd, then $\beta_{1}^{\prime}\left(K_{1, n} \cup K_{1, m}\right)=1$ by Theorem 2.3. But $K_{1, n} \cup K_{1, m}+e=$ $D S_{m, n}$, where $e$ joint two stars $K_{1, m}$ and $K_{1, n}$. Hence $\beta_{1}^{\prime}\left(D S_{m . n}\right)=2$ and upper bound is occurred.

\section{Acknowledgments}

The authors would like to thank the referees for their helpful remarks which have contributed to improve the presentation of the article. 


\title{
REF EREN C ES
}

1. R. P. Anstee, A polynomial algorithm for b-matchings an alternative approach, Inform. Process. Lett. 24(3) (1987), 153-157.

2. G. Chartrand, L. Lesniak and Ping Zhang, Graphs and digraphs, Sixth Edition (Chapman and Hall, Boca Raton, 2015).

3. A.N. Ghameshlou, A. Khodkar, R. Saei and S. M. Sheikholeslami, Signed (b, $k$ )- Edge Covers in Graphs, Intelligent Information Management 2 (2010), 143-148.

4. C. Wang, The signed matching in graphs, Discuss. Math. Graph Theory, 28(3)(2008), 477-486.

5. C. Wang, The signed k-submatching in graphs, Graphs Combin. 29(6) (2013), 19611971.

6. C. Wang, Signed b-matchings and b-edge covers of strong product graphs, Contrib. Discrete Math. 5(2) (2010), 1-10.

\author{
Samane Javan \\ Department of Mathematics \\ Science and Research Branch, Islamic Azad University \\ Tehran, Iran \\ javan.samane@gmail.com \\ Hamid Reza Maimani \\ Mathematics Section, Department of Basic Sciences \\ Shahid Rajaee Teacher Training University \\ P.O. Box 16785-163 \\ Tehran, Iran \\ maimani@ipm.ir
}

